\title{
Out-of-Plane and In-Plane Magnetization Behavior of Dipolar Interacting FeNi Nanoislands around the Percolation Threshold
}

\author{
A. Stupakov, ${ }^{1}$ A. V. Bagdinov, ${ }^{2}$ V. V. Prokhorov, ${ }^{3}$ A. N. Bagdinova, ${ }^{2}$ E. I. Demikhov, ${ }^{2}$ \\ A. Dejneka, ${ }^{1}$ K. I. Kugel, ${ }^{4}$ A. A. Gorbatsevich, ${ }^{2}$ F. A. Pudonin, ${ }^{2}$ and N. N. Kovaleva ${ }^{1,2,5}$ \\ ${ }^{1}$ Institute of Physics, ASCR, Na Slovance 2, 18221 Prague, Czech Republic \\ ${ }^{2}$ Lebedev Physical Institute, RAS, Leninsky Prosp. 53, Moscow 119991, Russia \\ ${ }^{3}$ Frumkin Institute of Physical Chemistry and Electrochemistry, RAS, Leninsky Prosp. 31, Moscow 119991, Russia \\ ${ }^{4}$ Institute for Theoretical and Applied Electrodynamics, RAS, Izhorskaya Str. 13, Moscow 125412, Russia \\ ${ }^{5}$ Department of Physics, Loughborough University, Leicestershire LE11 3TU, UK
}

Correspondence should be addressed to N. N. Kovaleva; n.kovaleva@lboro.ac.uk

Received 17 June 2016; Revised 16 September 2016; Accepted 19 October 2016

Academic Editor: Jean M. Greneche

Copyright (C) 2016 A. Stupakov et al. This is an open access article distributed under the Creative Commons Attribution License, which permits unrestricted use, distribution, and reproduction in any medium, provided the original work is properly cited.

\begin{abstract}
Magnetic properties of inhomogeneous nanoisland FeNi films were studied by SQUID magnetometry. The FeNi films with nominal thickness ranging from 0.6 to $2.0 \mathrm{~nm}$ were deposited by rf sputtering on Sitall glass substrates and covered by a protecting $\mathrm{Al}_{2} \mathrm{O}_{3}$ layer on the top. The SQUID data indicate pronounced irreversibility behavior for the out-of-plane temperature-dependent magnetization response (measured at $H \simeq 100 \mathrm{Oe}$ ) using zero-field cooling (ZFC) and field-cooled warming (FCW) after the applied dc magnetizing field $H_{m} \simeq 2 \mathrm{~T}$ for the FeNi samples with nominal thickness $1.1 \mathrm{~nm} \lesssim d \leqslant 1.8 \mathrm{~nm}$, below the percolation threshold. The positive difference between the FCW and ZFC data identifies two irreversibility temperature scales, $T_{B} \approx 50 \mathrm{~K}$ and $T^{*} \approx 200 \mathrm{~K}$, which can be associated with the superparamagnetic and superferromagnetic behavior in inhomogeneous nanoisland FeNi films, respectively. However, above the film percolation threshold, we observed a crossover from the out-of-plane to in-plane magnetization orientation. Here, the in-plane FCW-ZFC difference implies negative remanent magnetization response in the temperature range $T_{B} \lesssim T \lesssim T^{*}$. The observed magnetization properties can be associated with the presence of the superferromagnetic phase in self-assembled clusters of quasi-2D metallic magnetic FeNi nanoislands.
\end{abstract}

\section{Introduction}

Arrays of magnetic nanoparticles (dipolar magnets) are considered to form the basis of novel ultrahigh density magnetic data storage technology [1-3]. In dipolar magnets, each magnetic nanoparticle (NP) is in a single-domain ferromagnetic (FM) state with parallel orientation of intraparticle atomic moments arising due to strong exchange interactions. If the temperature is high enough to overcome energy barrier between different orientations of NP net magnetic moments, they exhibit the Curie-like behavior above the blocking temperature $\left(T_{B}\right)$ in the so-called superparamagnetic (SPM) phase. Meanwhile, owing to strong long-range dipolar interactions, the arrays of NPs can possess magnetic ordering at comparatively high temperatures, and such regime is called superferromagnetic (SFM). Indeed, dipolar interactions of single-domain NPs comprising many atomic moments $\left(\sim 10^{3} \div 10^{5} \mu_{B}\right)$ can be much stronger than ordinary dipolar interactions of localized atomic moments $\sim \mu_{B}$, where $\mu_{B}=$ $9.27 \cdot 10^{-21}$ emu is the Bohr magneton, which have a characteristic scale much less than $1 \mathrm{~K}$. For example, one can estimate that magnetic moment $m$ of $\mathrm{Fe}_{21} \mathrm{Ni}_{79}$ permalloy nanodiscs with diameter $a \sim 10 \div 30 \mathrm{~nm}$ and height $1 \mathrm{~nm}$ varies from $6.8 \cdot 10^{3}$ to $6.1 \cdot 10^{4} \mu_{B}$ (here, the saturation magnetization $M_{S} \simeq$ $\left.800 \mathrm{emu} / \mathrm{cm}^{3}\right)$. Then, the characteristic energy of dipoledipole interactions, $E_{\text {dip }}=2 \mathrm{~m}^{2} / \mathrm{r}^{3}$, of two similar singledomain nanodiscs located at the distance $r=30 \mathrm{~nm}$ varies from 2 to $180 \mathrm{~K}$.

In the SFM phase, the magnetic structure in regular arrays of single-domain NPs depends essentially on the lattice type and on the particle magnetic anisotropy. The latter, in turn, 
strongly depends on the particle shape and size. For example, in small enough nanoplatelets, spin reorientation transition (SRT) may lead to effective perpendicular magnetic anisotropy [4]. For 2D lattices of NPs with high perpendicular anisotropy, the magnetic structure corresponds to various types of two-sublattice antiferromagnetic (AF) order. For square lattices with in-plane anisotropy, the magnetic structure has four-sublattice AF order, whereas, for the triangular lattices, a FM order is implemented [5]. Here, for finite fragments of triangular lattices, the ground state can be a vortex state formed by the magnetic moments lying in the plane (supervortex) [6, 7]. Similarly, for soft magnetic dots [8-11], supervortex represents a topologically nontrivial ordered state, more intricate than the standard domain structure.

Magnetic properties in quasi-2D systems of inhomogeneously distributed magnetic NPs usually exhibit complex behavior as a consequence of the SPM and SFM phase coexistence [12]. Here, local magnetic order determined by geometric self-arrangements of neighboring interacting NPs can exist. In quasi-2D systems of inhomogeneous dipolar magnets, fairly separated weakly interacting NPs determine SPM behavior above blocking temperature $T_{B}$, whereas strongly interacting NPs in the close-packed assemblies are responsible for SFM behavior. By using electron holography with subparticle resolution, local in-plane FM versus AF order was observed in assemblies of $\sim 15 \mathrm{~nm}$ Co nanoparticles, depending on the close-packed triangular versus square arrangements, and even several flux-closed regions, which can be associated with a supervortex state, were recognized [13]. These results are supported by the numerical simulations, which emphasize that local dipolar magnetism in quasi-2D inhomogeneous NP systems survives even at a pronounced structural disorder [13].

Here, by using SQUID magnetometry, we study inplane and out-of-plane magnetic response in inhomogeneous nanoisland FeNi films, composed of flat nanoislands with lateral sizes of $5 \div 30 \mathrm{~nm}$, with the nominal film thickness varying from 0.6 to $2.0 \mathrm{~nm}$ (as schematically illustrated by Figure 1). The inhomogeneous FeNi films were grown by rf sputtering deposition on Sitall glass substrates, commonly used as film substrates in microelectronics. The experimental notice presented in [14], where equatorial magnetooptical Kerr effect was studied in periodic structures of alternating $\mathrm{FeNi}$ and Co nanoisland layers, suggests that a supervortex collective state is essentially relevant here. We present the evidence of the out-of-plane SFM magnetization behavior for the FeNi films with the nominal film thickness $1.1 \mathrm{~nm} \lesssim d \leqslant 1.8 \mathrm{~nm}$, below the physical percolation threshold. Recently, the outof-plane SFM behavior in quasi-2D Fe $(2.5 \mathrm{~nm}) / \mathrm{Al}_{2} \mathrm{O}_{3}$ multilayer composites was reported [12]. Moreover, we found a crossover from the out-of-plane to in-plane magnetization orientation with increasing nominal FeNi film thickness across the physical percolation threshold and discovered negative remanent magnetization response. This is in agreement with the earlier observation of negative remanence in nearly percolating magnetic granular $(\mathrm{Ni}, \mathrm{Fe})$ films in an insulating amorphous $\mathrm{SiO}_{2}$ matrix by Yan and $\mathrm{Xu}$ [15]. The understanding of the found out-of-plane and in-plane magnetization

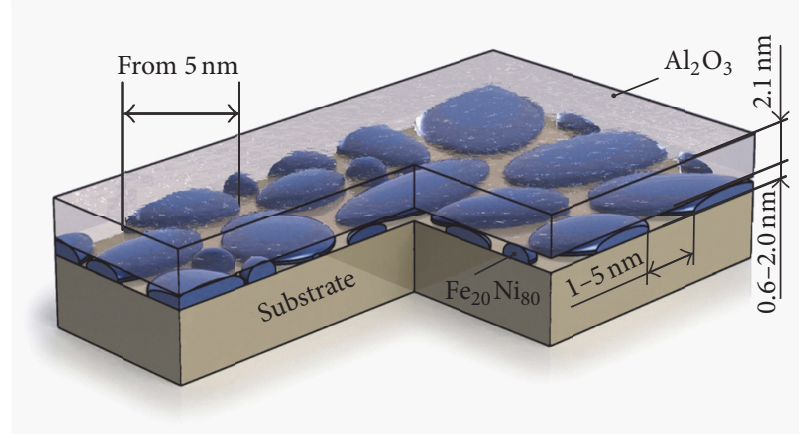

FIGURE 1: A schematic picture of the nanoisland FeNi film samples (capping $\mathrm{Al}_{2} \mathrm{O}_{3}$ layer $(2.1 \mathrm{~nm}) / \mathrm{FeNi}(d) /$ Sitall substrate).

properties, associated with self-organized ensembles of quasi2D single-domain FM FeNi nanoislands, is quite important for the fundamental physics of magnetism, as well as for technological applications.

\section{Materials and Methods}

2.1. FeNi Films Growth and Characterization. The nanoisland $\mathrm{FeNi}$ films were grown by $\mathrm{rf}$ sputtering deposition from $\mathrm{Fe}_{21} \mathrm{Ni}_{79}$ targets at a base vacuum pressure less than $2 \times$ $10^{-6}$ Torr and a background argon pressure of $4 \times 10^{-4}$ Torr. Glass-like Sitall material was used for substrates. The analysis of an X-ray diffraction pattern of the used Sitall substrate showed that it is represented by $\mathrm{TiO}_{2}$ rutile phase [16]. During the deposition, the substrate temperature was $73 \pm 3^{\circ} \mathrm{C}$. The nominal film thickness (i.e., the thickness of the corresponding continuous film) was controlled by the deposition rate and time (see more details in [17]). We prepared the nanoisland FeNi films with nominal thickness varying from 0.6 to $2.0 \mathrm{~nm}$. Our earlier spectroscopic ellipsometry studies of the nanoisland FeNi films of different thickness grown on the Sitall glass demonstrated that their dielectric permittivity changes from insulating-like to metallic-like at the nominal FeNi film thickness about $1.8 \mathrm{~nm}$. In addition, the temperature dependence of dc conductivity suggests the existence of the percolation threshold for the same nominal FeNi film thickness [18]. To avoid oxidation of the films at ambient conditions, the grown FeNi films were covered in situ by the $\mathrm{Al}_{2} \mathrm{O}_{3}$ capping layer $2.1 \mathrm{~nm}$ thick.

\subsection{Atomic-Force Microscopy Study of the Grown FeNi Films.} Surface morphology of the nanoisland FeNi films grown by the rf sputtering deposition on the Sitall glass substrates was studied by atomic-force microscopy (AFM) using Ntegra Prima (NT-MDT, Zelenograd, Russia) facility. Figure 2(a) represents a large-scale AFM image of the FeNi film sample $\left(\mathrm{Al}_{2} \mathrm{O}_{3}(2.1 \mathrm{~nm}) / \mathrm{FeNi}(d) /\right.$ Sitall substrate $)$ with the nominal film thickness $d \simeq 1.2 \mathrm{~nm}$. The shown large-scale topography profile indicates the height variation in the range $1 \div 3 \mathrm{~nm}$, which characterizes the surface roughness. The smaller-size image in Figure 2(b) clearly identifies the grainy structure with the grain lateral dimensions in the range of $15 \div 25 \mathrm{~nm}$. 


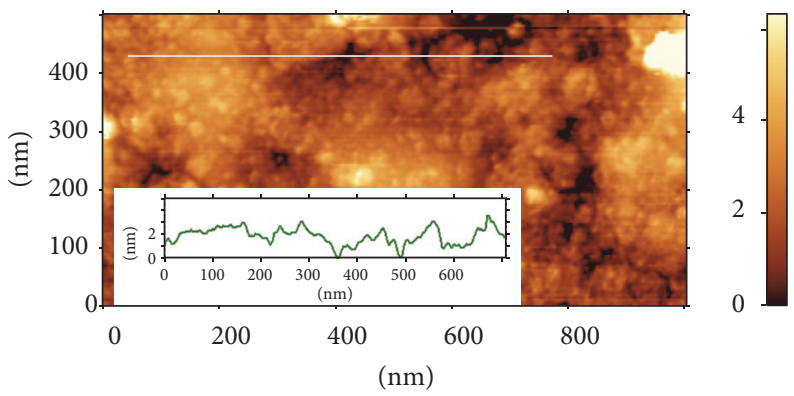

(a)

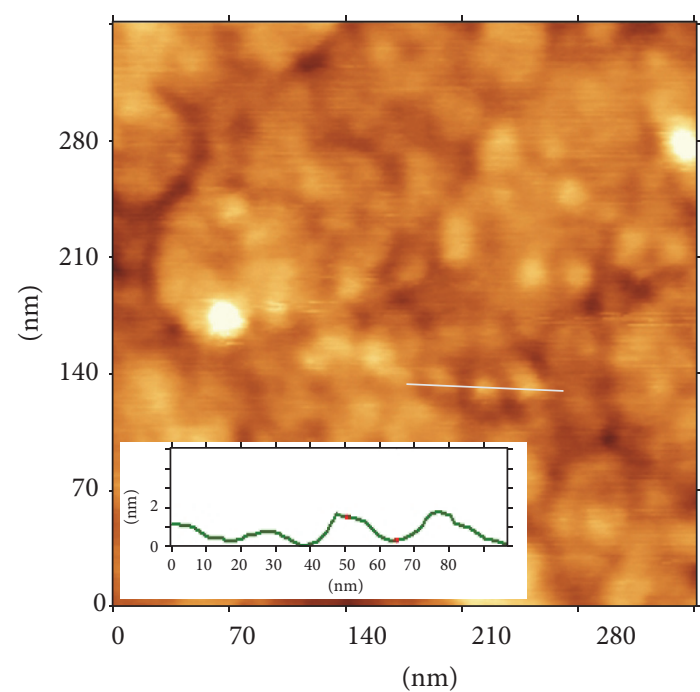

(b)
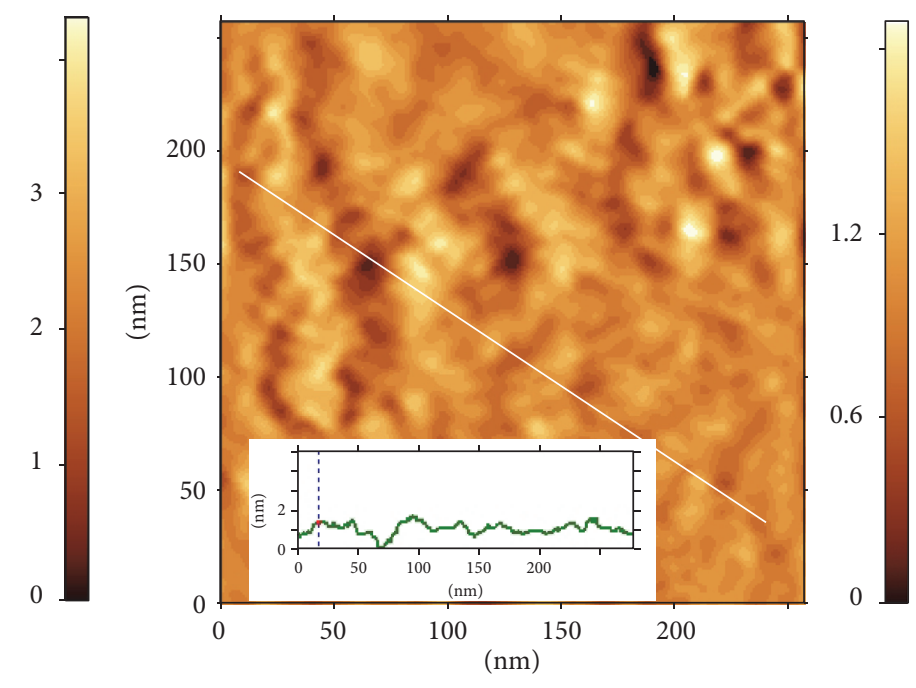

(c)

FIGURE 2: AFM images of the FeNi film samples $\left(\mathrm{Al}_{2} \mathrm{O}_{3}(2.1 \mathrm{~nm}) / \mathrm{FeNi}(d) /\right.$ Sitall substrate $)$ with the nominal film thickness $(\mathrm{a}, \mathrm{b}) d \simeq 1.2 \mathrm{~nm}$ and (c) $d \simeq 1.9 \mathrm{~nm}$.

Due to intrinsically uneven surface of the substrate, the grain height fluctuates strongly. The typical height is of about $1 \mathrm{~nm}$ (see the height profile in Figure 2(b)). The inevitable AFM broadening does not allow estimating the real width of gaps between the grains. Figure 2(c) represents an AFM image of the FeNi film sample $\left(\mathrm{Al}_{2} \mathrm{O}_{3}(2.1 \mathrm{~nm}) / \mathrm{FeNi}(d) /\right.$ Sitall substrate) with the nominal film thickness $d \simeq 1.9 \mathrm{~nm}$. Due to appreciable coalescence of the nanoislands in the film with the thickness above the percolation threshold at $d_{c} \simeq 1.8 \mathrm{~nm}$ [18], the topography profile is more shallow in the percolating regions.

2.3. SQUID Measurements. For magnetization measurements, we cut out the FeNi film samples of approximate dimensions $3 \times 3 \mathrm{~mm}^{2}$. Using the SQUID magnetometer MPMS $\mathrm{XL} 7 \mathrm{~T}$, we were able to measure magnetization in the temperature range from 2 to $300 \mathrm{~K}$. High sensitivity of magnetic measurements $\left(2 \times 10^{-8} \mathrm{emu}\right)$ was enabled by reciprocating sample transport. Recently, by using a MPMS XL 7 T SQUID magnetometer, total magnetization of $d^{0}$ charge-imbalanced FM interface between nonmagnetic perovskites of the order of $10^{-6}$ emu was reported [19]. For the zero-field-cooled (ZFC) measurements, a sample was first cooled down to
$T \simeq 5 \mathrm{~K}$ in zero magnetic field. Then, the magnetic field $H \simeq 100$ Oe was applied, and the ZFC data were collected, while the sample is slowly warmed up above the irreversibility temperature. We would like to point out that, for singledomain $\mathrm{Fe}_{21} \mathrm{Ni}_{79}$ nanoislands, the stray magnetic fields at the edge constitute $1.08 \mathrm{~T}$. Therefore, to avoid uncertainties in the determination of the equilibrium magnetization at small $H$ and to achieve a fully polarized magnetic state, we applied the large magnetizing filed $H_{m} \simeq 2 \mathrm{~T}$, while the film sample was cooled down to $5 \mathrm{~K}$, finally switching the field off. Then, the field-cooled warming (FCW) data were collected, while the sample is slowly warmed up in the same measurement field $H \simeq 100$ Oe. In a more usual protocol, after the ZFC part, a second set of data is collected, while the sample is slowly cooled down in the same field, the field-cooled (FC) part. However, use of the ZFC-FC protocol, when the measurement field is not large, usually of about $100 \div 200$ Oe, cannot guarantee a fully polarized magnetic state in our case.

\section{Results and Discussion}

Figures 3(a)-3(d) show the ZFC and FCW magnetization response of the FeNi film samples (schematically illustrated by Figure 1) with different nominal film thickness varying 


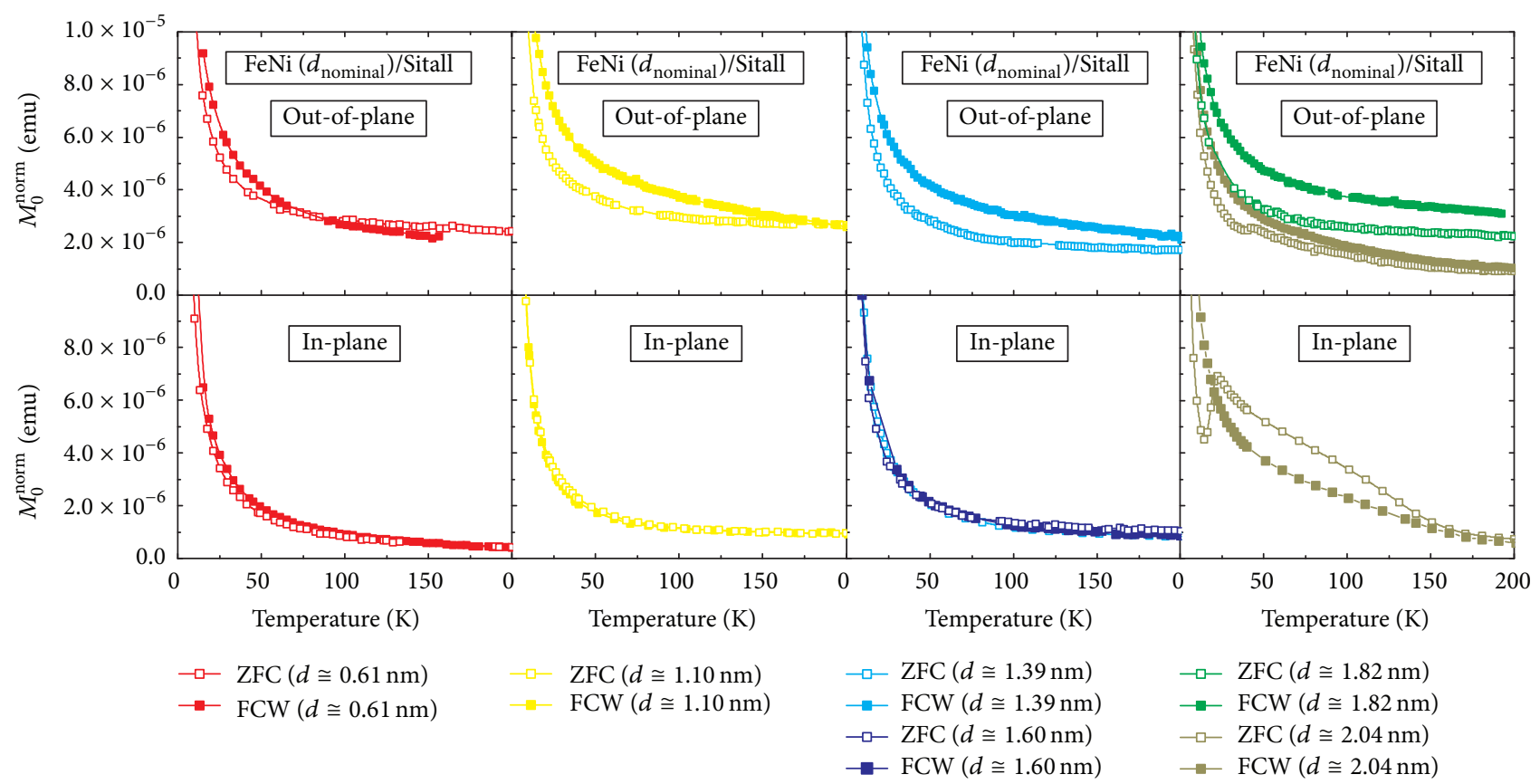

(a)

(b)

(c)

(d)

FIGURE 3: The out-of-plane (top panels) and in-plane (bottom panels) ZFC and FCW normalized magnetization response (calculated using (1)) of the nanoisland FeNi film samples $\left(\mathrm{Al}_{2} \mathrm{O}_{3}(2.1 \mathrm{~nm}) / \mathrm{FeNi}(d) /\right.$ Sitall substrate) with the nominal film thickness of (a) $0.61 \mathrm{~nm},(\mathrm{~b}) 1.10 \mathrm{~nm}$, (c) 1.39 and $1.60 \mathrm{~nm}$, and (d) 1.82 and $2.04 \mathrm{~nm}$. The displayed symbols are larger than the error bars. The solid curves are the guides to the eye.

from 0.61 to $2.04 \mathrm{~nm}$, registered in the in-plane and out-ofplane geometry of the applied magnetic field. To compare the ZFC and FCW magnetization response, $M_{0}(T)$, measured for the different FeNi film samples, the magnetization response was normalized to the equal sample area, $S=10^{-1} \mathrm{~cm}^{2}$ (which is typical for our samples measured here by SQUID magnetometry), using the following formula:

$$
M_{0}^{\text {norm }}(T) \simeq S \rho_{S} L \frac{M_{0}(T)}{m_{0}},
$$

where $m_{0}$ is the sample mass, $\rho_{S} \simeq 2.72 \pm 0.08 \mathrm{~g} / \mathrm{cm}^{3}$ is the Sitall substrate density, and $L \simeq 0.056 \pm 0.004 \mathrm{~cm}$ is the substrate thickness. The normalized magnetization response of the FeNi film samples shown in Figures 3(a)-3(d) is typical paramagnetic-like, due to the dominating contribution of the Sitall substrate, which contains clustered magnetic impurities and/or defects. We fitted the in-plane magnetization field dependence of the Sitall substrate measured at $T \simeq 10 \mathrm{~K}$ with the Langevin function $M(H, T)=N_{p} \mu_{p}\left[\operatorname{coth}\left(\mu_{p} H / k_{\mathrm{B}} T\right)-\right.$ $\left.k_{\mathrm{B}} T / \mu_{p} H\right]$, where $k_{\mathrm{B}}$ is Boltzmann's constant and estimated average magnetic moment of magnetic impurities $\mu_{p} \simeq 6 \mu_{B}$ and their concentration $N_{p} \simeq 2.03 \cdot 10^{19} \mathrm{~cm}^{-3}$.

One can notice that the out-of-plane magnetization response, $M_{0}^{\text {norm }}(T)$, clearly demonstrates difference between the FCW and ZFC curves for all studied samples (see the top panels of Figures 3(a)-3(d)). However, we found that the FCW-ZFC difference is remarkably pronounced for the FeNi film samples with the nominal film thickness $1.1 \mathrm{~nm} \leqslant d \leqslant$ $1.8 \mathrm{~nm}$, where the associated irreversibility behavior persis- ted up to $T^{*} \approx 200 \mathrm{~K}$ (see Figures $3(\mathrm{~b})-3(\mathrm{~d})$ and $4(\mathrm{~b})$ ). In addition, a clear kink was observed there for the out-ofplane FCW-ZFC difference at $T_{B} \approx 50 \mathrm{~K}$ (as illustrated by Figure $4(\mathrm{~b})$ ). The revealed temperatures, $T_{B} \approx 50 \mathrm{~K}$ and $T^{*} \approx$ $200 \mathrm{~K}$, indicate the existence of two different temperature scales for the out-of-plane irreversibility behavior of the studied nanoisland FeNi films. We noticed an apparent analogy to the results for the quasi-2D Fe $(2.5 \mathrm{~nm}) / \mathrm{Al}_{2} \mathrm{O}_{3}$ multilayer composites at low-filling factor [12]. In line with [12], the temperatures $T_{B} \approx 50 \mathrm{~K}$ and $T^{*} \approx 200 \mathrm{~K}$ can be associated with the SPM and SFM behavior, respectively. Here, fairly separated and weakly interacting small FeNi nanoislands determine the SPM behavior above the blocking temperature $T_{B} \approx$ $50 \mathrm{~K}$, whereas strongly interacting FeNi nanoislands in their dispersive assemblies are responsible for the induced out-ofplane SFM behavior. The latter is indicated by the additional hysteretic-like contribution persistent up to the higher irreversibility temperature $T^{*} \approx 200 \mathrm{~K}$ (as it is schematically illustrated by Figure 5(b)). From Figure 4(b), one can estimate that the SFM hysteretic-like contribution attains its maximum of about $1.8 \cdot 10^{-6}$ emu at low temperatures $(\approx 20 \%$ from the saturation magnetization of $\mathrm{Fe}_{21} \mathrm{Ni}_{79}$ permalloy, here one should take into account that saturation magnetization of NPs is usually somewhat less than that of a bulk material).

Analyzing the in-plane normalized magnetization response, $M_{0}^{\text {norm }}(T)$, obtained from the FCW and ZFC measurements, we can distinguish three different types of behavior, depending on the nominal FeNi film thickness (see the bottom panels of Figures 3(a)-3(d) and Figures 4(a)-4(c)). Thus, for the thinnest investigated FeNi film sample (i) with 


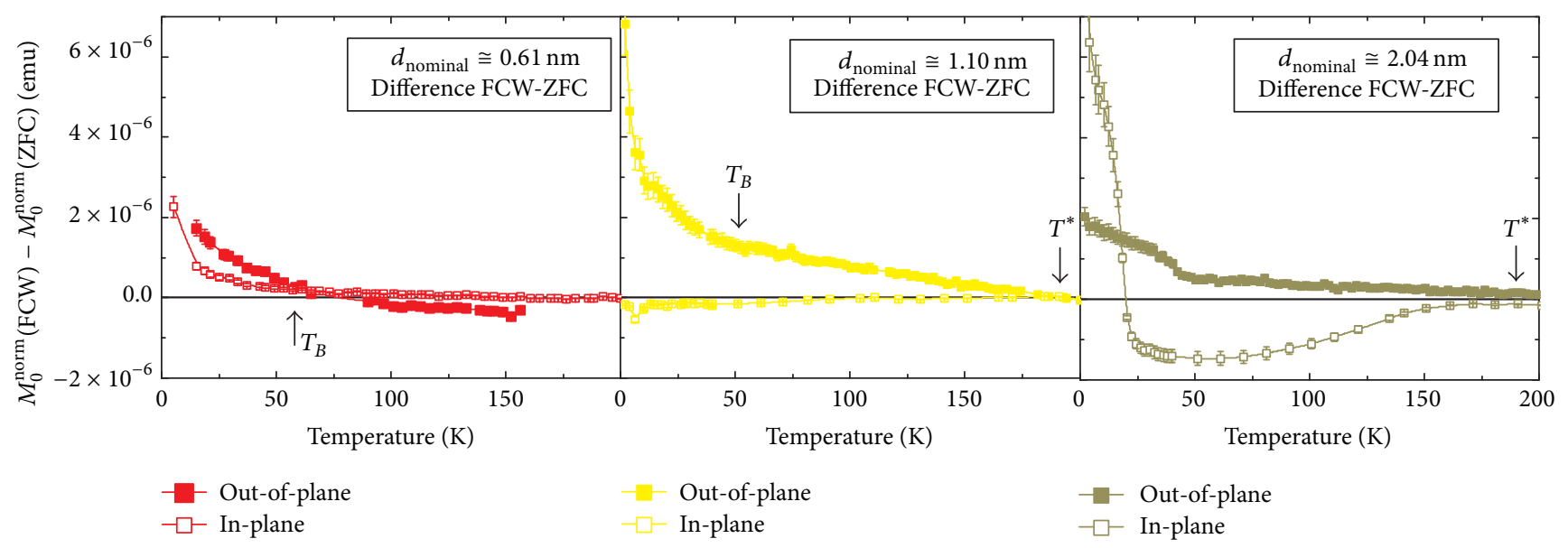

(a)

(b)

(c)

Figure 4: The difference between the FCW and ZFC normalized magnetization response shown in Figure 3 for the FeNi film samples $\left(\mathrm{Al}_{2} \mathrm{O}_{3}\right.$ $(2.1 \mathrm{~nm}) / \mathrm{FeNi}(d) /$ Sitall substrate) with the nominal film thickness (a) $0.61 \mathrm{~nm}$, (b) $1.10 \mathrm{~nm}$, and (c) $2.04 \mathrm{~nm}$ in the out-of-plane and in-plane sample configuration. The solid curves are the guides to the eye.

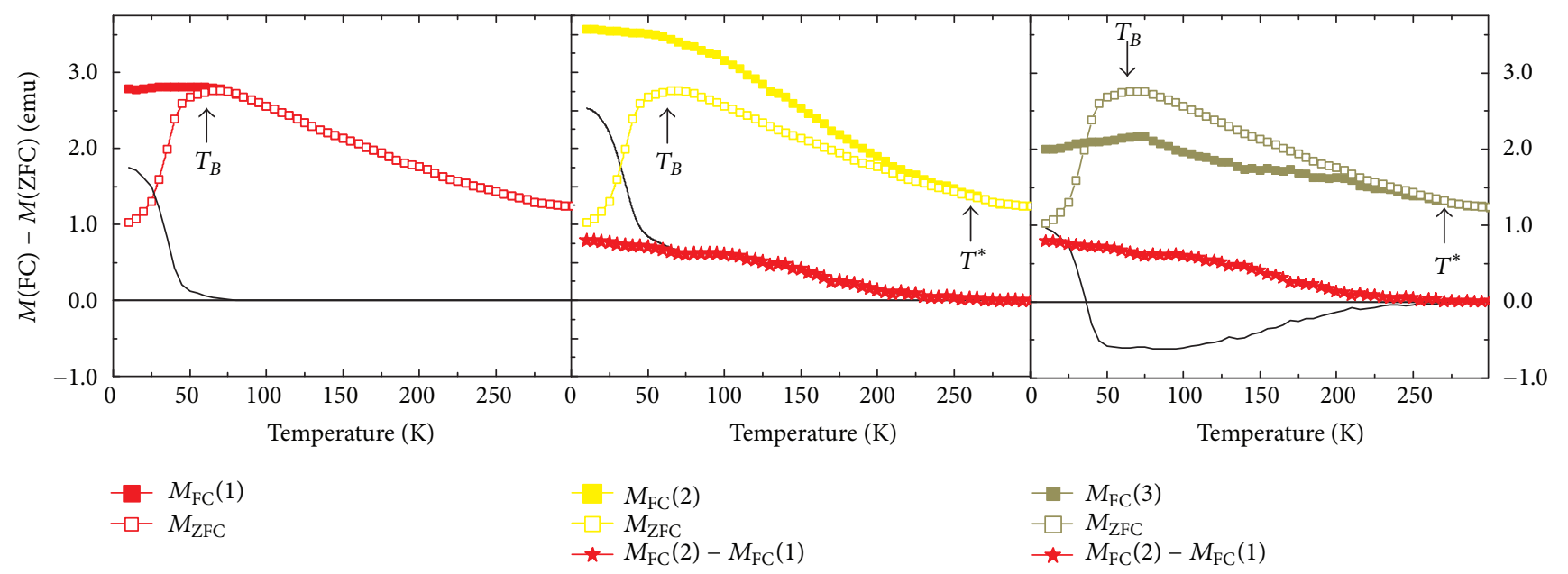

(a)

(b)

(c)

FIGURE 5: A schematic illustration of the temperature-dependent ZFC and FC magnetization response (and of the associated difference, shown by a solid black line) for FM NPs. (a) For SPM phase of weakly interacting NPs. (b) For SPM phase of weakly interacting NPs, coexisting with SFM phase of strongly interacting NPs. Here, red star symbols correspond to the additional hysteretic-like SFM contribution. (c) Modeling the in-plane negative response (shown in Figure 4(c)) above the NP's system percolation threshold. Here, the FC curve is obtained by subtraction of the SFM contribution, $\mathrm{M}_{\mathrm{FC}}(3)=\mathrm{M}_{\mathrm{FC}}(1)-\left[\mathrm{M}_{\mathrm{FC}}(2)-\mathrm{M}_{\mathrm{FC}}(1)\right]$.

the nominal thickness $d \simeq 0.61 \mathrm{~nm}$, the FCW-ZFC difference is quite small (see Figure 3(a)). Here, the in-plane FCW-ZFC difference starts to deviate from nearly zero values (within the experimental accuracy) below $\approx 60 \div 70 \mathrm{~K}$ (see Figure $4(\mathrm{a})$ ), indicating the associated SPM character of the irreversibility behavior below the blocking temperature (as it is schematically demonstrated by Figure 5(a)). The normalized FCWZFC difference attains its maximum of about $2 \cdot 10^{-6} \mathrm{emu}$ at $5 \mathrm{~K}\left(\approx 45 \%\right.$ from the saturation magnetization of $\mathrm{Fe}_{21} \mathrm{Ni}_{79}$ permalloy) (see Figure 4(a)).

However, for the studied FeNi film samples (ii) with nominal thickness in the range $1.1 \mathrm{~nm} \leqslant d \leqslant 1.8 \mathrm{~nm}$, the inplane response did not show clearly perceptible difference between the temperature-dependent magnetization response using $\mathrm{ZFC}$ and FCW at the dc magnetizing field $H_{m} \simeq 2 \mathrm{~T}$ (see Figures 3(b), 3(c), and 4(b)).

And finally, there is a remarkable difference between the in-plane FCW and ZFC normalized magnetization curves for the FeNi film sample (iii) with the thickness $d \simeq 2.04 \mathrm{~nm}$, above the physical percolation threshold at $d_{c} \simeq 1.8 \mathrm{~nm}$ [18] (see the bottom panel of Figure 3(d)). Here, the in-plane ZFC curve shows a pronounced maximum at the blocking temperature $T_{B}^{c} \simeq 25 \mathrm{~K}$. This implies the existence of a crossover from the out-of-plane to in-plane magnetization orientation. $T_{B}^{c} \simeq$ $25 \mathrm{~K}$ seemingly manifests SPM properties in the FeNi film close to the percolation threshold at $d_{c} \simeq 1.8 \mathrm{~nm}$ [18]. Below 


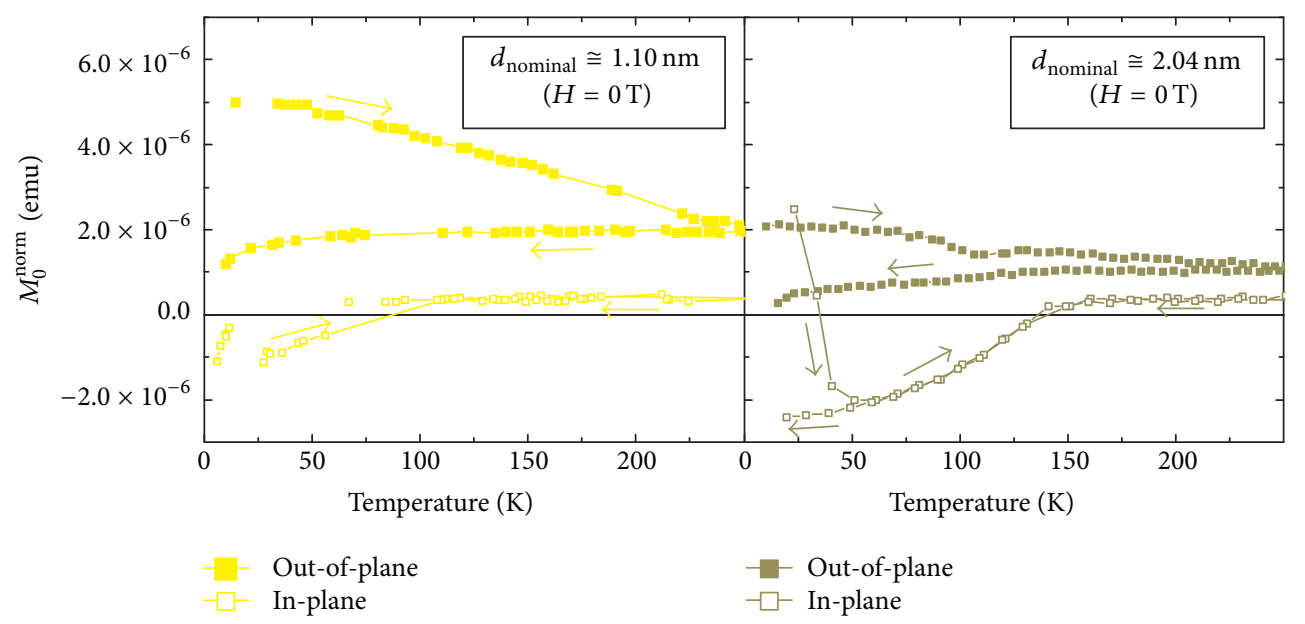

(a)

(b)

FIGURE 6: The temperature dependence of the normalized remanent magnetization, produced by the in-plane and out-of-plane magnetizing field $H_{m} \simeq 1 \mathrm{~T}$ at $T \simeq 10 \mathrm{~K}$ in the studied FeNi film samples $\left(\mathrm{Al}_{2} \mathrm{O}_{3}(2.1 \mathrm{~nm}) / \mathrm{FeNi}(d) /\right.$ Sitall substrate) with the nominal film thickness (a) $1.10 \mathrm{~nm}$ and (b) $2.04 \mathrm{~nm}$. Arrows indicate the magnetization response $(H \simeq 0)$ with cycling temperature from $10 \mathrm{~K}$ to $300 \mathrm{~K}$ and back, down to low temperatures. The displayed symbols are larger than the error bars. The solid curves are the guides to the eye.

$T_{B}^{c}$, the normalized FCW-ZFC difference attains its maximum of about $6 \cdot 10^{-6} \mathrm{emu}$ at $10 \mathrm{~K}(\approx 37 \%$ from the saturation magnetization of $\mathrm{Fe}_{21} \mathrm{Ni}_{79}$ permalloy) (see Figure 4(c)). Surprisingly, here, the in-plane FCW curve exhibits lower magnetization values than that of the corresponding ZFC curve from about $T_{B}^{c}$ to $T^{*} \approx 200 \mathrm{~K}$ (see the bottom panel of Figure 3(d)). Due to this, the normalized FCW-ZFC difference turns out to be negative, with a pronounced minimum of about $-1.8 \cdot 10^{-6}$ emu around $50 \mathrm{~K}$ (see Figure 4(c)). And, with rising temperature above the SFM irreversibility temperature $T^{*} \approx 200 \mathrm{~K}$, the FCW-ZFC difference vanishes. In contrast to the induced out-of-plane SFM behavior found below the percolation threshold, where the FCW curve lies above the ZFC curve, here, the FCW curve lies below the ZFC curve. According to our simple modeling, this behavior can be adjusted by the negative SFM contribution (as it is schematically illustrated by Figure 5(c)). The found behavior indicates that the magnetization response is in a direction opposite to the applied dc magnetizing field $H_{m} \simeq 2 \mathrm{~T}$ (while the film sample was cooled down from above $T^{*} \approx 200 \mathrm{~K}$ to $5 \mathrm{~K}$ ), and it can be associated with the SFM component.

Figures 6(a) and 6(b) display temperature dependence of the in-plane and out-of-plane normalized remanent magnetization response of the nanoisland $\mathrm{FeNi}$ film samples (capping $\mathrm{Al}_{2} \mathrm{O}_{3}$ layer $(2.1 \mathrm{~nm}) / \mathrm{FeNi}$ film (d)/Sitall substrate) produced by the magnetizing field $H_{m} \simeq 1 \mathrm{~T}$ at $10 \mathrm{~K}$. Here, after the magnetizing field was switched off, the remanent magnetization was recorded at zero external magnetic field $(H \simeq 0 \mathrm{~T})$ on rising temperature from 10 to $300 \mathrm{~K}$ and then on cooling down to the lowest measuring temperature. The magnetization measurements at $H \simeq 0 \mathrm{~T}$ allowed us to get rid of the paramagnetic-like contribution of the Sitall substrate (dominating the magnetization response shown in Figures $3(a)-3(d))$. One can notice from Figure 6(a) that the outof-plane remanent magnetization of the FeNi film with the thickness $d \simeq 1.1 \mathrm{~nm}$ decreases from about $5 \cdot 10^{-6} \mathrm{emu}$ at $10 \mathrm{~K}\left(\approx 57 \%\right.$ from the saturation magnetization of $\mathrm{Fe}_{21} \mathrm{Ni}_{79}$ permalloy) to about $2 \cdot 10^{-6} \mathrm{emu}$ at $300 \mathrm{~K}(\approx 23 \%$, resp.). The observed trends of the in-plane remanent magnetization are the most pronounced for the FeNi film sample with the nominal thickness $d \simeq 2.04 \mathrm{~nm}$ (see Figure 6(b)). Here, the FeNi film was initially magnetized along the magnetic field $\left(H_{m} \simeq\right.$ $1 \mathrm{~T}$ ) direction applied in the film plane at $10 \mathrm{~K}$. Surprisingly, with rising temperature up to $50 \mathrm{~K}$, the in-plane normalized remanent magnetization relaxed down to negative values of about $-2 \cdot 10^{-6}$ emu. Here, above the blocking temperature $T_{B}$, large magnetic moments of individual SPM nanoislands become strongly fluctuating, and the resulting contribution from the SPM component is zero at $H \simeq 0$. This means that some parts of the FeNi film, related to the SFM component, retain the magnetization opposite to the applied magnetizing field $H_{m} \simeq 1 \mathrm{~T}$. Indeed, with further increasing temperature above the irreversibility temperature $T^{*}$, the negative magnetization response disappeared, and the in-plane remanent magnetization attained a small value at $300 \mathrm{~K}$. Interestingly, the in-plane temperature dependence of the magnetization was reproducible at subsequent cooling down, exhibiting the same negative magnetization response below $150 \mathrm{~K}$ down to $50 \mathrm{~K}$. This means that the negative magnetization component is related to a magnetically ordered ground state of the SFM phase. It remains intact to the applied magnetizing field $H_{m} \simeq$ $1 \mathrm{~T}$, preserving the initial magnetization orientation. The result shown in Figure 6(b) suggests that, around $50 \mathrm{~K}$, the inverted hysteresis $M-H$ loop will be observed for the inplane measurements, with the negative magnetization value of $-12 \%$ at $H \simeq 0$ (normalized to positively saturated value). One can notice from Figure 6(a) that the temperature dependence of the in-plane remanent magnetization of the $\mathrm{FeNi}$ film with the nominal thickness $d \simeq 1.1 \mathrm{~nm}$ showed, in main, similar trends. However, the observed temperature effects 
were less pronounced here and shifted to lower temperatures. We note that the observed trends of the remanent magnetization have a strong resemblance to the results of the independent FCW-ZFC measurements (see Figures 4(b) and 4(c)), giving supporting experimental evidence.

Now, we discuss the observed magnetization properties. The observed out-of-plane SFM behavior below the percolation threshold can be associated with an effective perpendicular magnetic anisotropy in the rf sputtered FeNi films composed of quasi-2D FM FeNi nanoislands. For example, an effective perpendicular anisotropy can exist in small enough quasi-2D FeNi nanoislands due to SRT [4]. As a result, the local magnetic structure may correspond to AF ground state on square or triangular lattice fragments of self-assembled quasi-2D FeNi nanoislands. In addition, here, we observed a crossover from the out-of-plane to in-plane magnetization orientation with increasing the nanoisland size close to the percolation transition. Here, the in-plane magnetic structure may have four-sublattice AF order for square lattice fragments, whereas a FM order may be implemented for the triangular lattice fragments.

We would like to note that such system with an effective perpendicular magnetic anisotropy has also a tendency to inhomogeneous distribution of magnetic moments in the form of supervortices [7]. We infer that the studied nanoisland FeNi films, composed of inhomogeneously distributed FM single-domain nanoislands, represent a unique playground to challenge a supervortex state and its magnetic properties. For small magnetic anisotropy, a purely planar vortex can exist on close-packed hexagonal fragments of a triangular lattice, with in-plane distribution of FM NP's magnetic moments. Here, the total out-of-plane projection of the magnetic moment vanishes, but the in-plane component of the total magnetization is nonzero, as the magnetic moment of the vortex core remains not compensated [7]. This may be relevant to the in-plane magnetization properties found in the present study for the nanoisland FeNi film with the nominal film thickness above the percolation threshold at $d_{c} \simeq 1.8 \mathrm{~nm}$. With the increase of the particle magnetic anisotropy, the vortex core starts to protrude out-of-plane. And with the further increase, the symmetry of the vortex ground state increases, where the planar magnetization component vanishes (featuring zero-net magnetic moment), but the perpendicular component changes significantly [7]. This may be well consistent with the magnetization properties found in the present study for the nanoisland FeNi films with the nominal film thickness $1.1 \mathrm{~nm} \lesssim d \lesssim 1.8 \mathrm{~nm}$.

Recently, the out-of-plane SFM behavior in quasi-2D Fe $(2.5 \mathrm{~nm}) / \mathrm{Al}_{2} \mathrm{O}_{3}$ multilayer composites was reported [12]. The results by Miu et al. indicate that the dipolar interactions are not the major interactions and support the relevance of twodimensionality and additional short-range "superexchange" interactions for the occurrence of the out-of-plane SFM behavior in quasi-2D FM NPs above some critical filling factor (see [12] and references therein). In particular, the stray fields of non-point-like magnetic dipoles can promote this "superexchange" and drive SFM order in quasi-2D FM NPs. In addition, an indirect Ruderman-Kittel-Kasuya-Yosida (RKKY) interaction between FM NPs in quasi-2D magnetic structures can contribute to the short-range "superexchange" coupling mechanism and be responsible for SFM behavior [20]. Thus, the SFM phase, associated with complex magnetic behavior in quasi-2D clusters of large NP's localized magnetic moments, implies essentially a Many-Body Localized (MBL) state [21].

In addition, here, we demonstrated that the discovered inplane negative magnetization response above the nanoisland FeNi film percolation threshold can be associated with the SFM component. Earlier, negative remanent magnetization was observed in nearly percolating magnetic granular $(\mathrm{Ni}$, $\mathrm{Fe}$ ) films in an insulating amorphous $\mathrm{SiO}_{2}$ matrix [15]. The temperature-dependent remanence magnetization observed there and shown in Figure 2 of [15] is similar to the in-plane magnetization behavior found in the present study for FeNi nanoislands above the percolation threshold (see Figures 4(c) and $6(\mathrm{~b}))$. The remanence observed in [15] was as large as $-9 \%$ compared to the positively saturated value. It was suggested in this study that near the percolation threshold the magnetostatic interaction between coexisting SPM and FM components, with a special geometry of the FM nanoclusters, favors their opposite alignment, induced by the applied magnetizing field. An alternative interpretation in terms of interface exchange interaction or exchange anisotropy was suggested for similar phenomena observed in amorphous and multilayered materials (see [15] and references therein). The negative remanent magnetization is possible in an exchange-coupled bilayer, when a magnetically soft material is influenced by the demagnetizing field of the hard material. Note that the observed phenomena have a strong similarity with exchange bias effect, actively studied in many composite magnetic materials.

\section{Conclusions}

We present the evidence of the out-of-plane SFM behavior for the nanoisland FeNi films with the nominal film thickness $1.1 \mathrm{~nm} \lesssim d \lesssim 1.8 \mathrm{~nm}$ below the percolation threshold at $d_{c} \simeq 1.8 \mathrm{~nm} \mathrm{[18]}$ in the temperature range, which fits well to the estimated characteristic energy of the long-range dipolar interactions of about $180 \mathrm{~K}$ (the estimate is given in Introduction). The SFM behavior is indicated by the additional hysteretic-like contribution persistent up to the irreversibility temperature $T^{*} \approx 200 \mathrm{~K}$. Besides, an admixture of the SPM phase was identified here by a clear kink in the out-of-plane FCW-ZFC difference at $T_{B} \simeq 50 \mathrm{~K}$.

Above the film percolation threshold, we observed a crossover from the out-of-plane to in-plane magnetization orientation. Here, the ZFC curve shows a clear maximum near the blocking temperature $T_{B}^{c} \simeq 25 \mathrm{~K}$, which certifies the presence of SPM component. The in-plane FCW-ZFC difference turns out to be negative in the temperature range $T_{B} \lesssim T \lesssim T^{*}$, implying that the magnetization response is in a direction opposite to the applied dc magnetizing field $H \simeq 2 \mathrm{~T}$. The investigation of $M(H)$ hysteresis loops at small applied magnetic fields at different temperatures will be interesting and relevant here. We showed that the discovered inplane negative magnetization response above the nanoisland FeNi film percolation threshold can be associated with the 
SFM component. From our study, we can conclude that the negative magnetization response can be related to some parts of the FeNi film, which retain the magnetization opposite to the applied magnetizing field, preserving their initial magnetization orientation. These parts, related to the SFM component, can be considered as being magnetically hard, so that the direction of their magnetization could not be changed by the applied magnetizing field. The origin of the magnetically hard component in the studied nanoisland FeNi films needs to be further investigated. For example, its relevance to a core of the purely planar vortex, which can exist on closepacked hexagonal fragments of a triangular lattice here, with in-plane distribution of FM NP's magnetic moments, should be challenged.

We conclude that the observed magnetization properties can be associated with the SFM behavior in self-assembled clusters of quasi-2D metallic magnetic FeNi nanoislands. The SFM phase associated with complex magnetic behavior in quasi-2D clusters of large NP's localized magnetic moments $\left(\sim 10^{3} \div 10^{5} \mu_{B}\right)$ implies a MBL state. The electronic excitations [21-23] of this MBL state and response to strong applied magnetic fields need to be further fundamentally studied. Also, the understanding of the found out-of-plane and in-plane SFM behavior, associated with self-organized ensembles of quasi-2D single-domain nanoislands, requires further studies by using, for example, magnetic imaging techniques.

\section{Abbreviations}

ZFC: Zero-field cooling
FC: $\quad$ Field cooling
FCW: Field-cooled warming
NP: Nanoparticle
FM: Ferromagnetic
SPM: Superparamagnetic
SFM: Superferromagnetic
SRT: Spin reorientation transition
AF: Antiferromagnetic
AFM: Atomic-force microscopy.

\section{Competing Interests}

The authors declare no competing financial interests.

\section{Acknowledgments}

The authors acknowledge fruitful discussions with M. Forrester, F. Kusmartsev, and N. Sibeldin. This work was supported by the Czech Science Foundation GA CR (Grant no. 15-13778S) and by the Russian Foundation for Basic Research (Projects 14-02-00276 and 16-02-00304). Our experiments were performed in MLTL (http://mltl.eu/), which is supported within the program of Czech Research Infrastructures (Project no. LM2011025).

\section{References}

[1] S. Sun and C. B. Murray, "Synthesis of monodisperse cobalt nanocrystals and their assembly into magnetic superlattices," Journal of Applied Physics, vol. 85, no. 8, pp. 4325-4330, 1999.

[2] G. Reiss and A. Hütten, "Magnetic nanoparticles: applications beyond data storage," Nature Materials, vol. 4, no. 10, pp. 725726, 2005.

[3] N. A. Frey and S. Sun, "Magnetic nanoparticle for information storage applications," in Inorganic Nanoparticles: Synthesis, Applications, and Perspectives, C. Altavilla and E. Ciliberto, Eds., chapter 3, pp. 33-68, CRC Press, New York, NY, USA, 2010.

[4] E. Y. Vedmedenko, H. P. Oepen, and J. Kirschner, "Size-dependent spin reorientation transition in nanoplatelets," Physical Review B-Condensed Matter and Materials Physics, vol. 67, no. 1, Article ID 012409, pp. 1-4, 2003.

[5] V. M. Rozenbaum, V. M. Ogenko, and A. A. Chulko, "Vibrational and orientational states of surface atomic groups," Soviet Physics Uspekhi, vol. 34, no. 10, pp. 883-902, 1991.

[6] P. Politi, M. G. Pini, and R. L. Stamps, "Dipolar ground state of planar spins on triangular lattices," Physical Review BCondensed Matter and Materials Physics, vol. 73, no. 2, Article ID 020405, pp. 1-4, 2006.

[7] S. A. Dzian, A. Y. Galkin, B. A. Ivanov, V. E. Kireev, and V. M. Muravyov, "Vortex ground state for small arrays of magnetic particles with dipole coupling," Physical Review B-Condensed Matter and Materials Physics, vol. 87, no. 18, pp. 1-7, 2013.

[8] M.-Y. Im, P. Fischer, K. Yamada et al., "Symmetry breaking in the formation of magnetic vortex states in a permalloy nanodisk," Nature Communications, vol. 3, pp. 983-988, 2012.

[9] R. P. Cowburn, D. K. Koltsov, A. O. Adeyeye, M. E. Welland, and D. M. Tricker, "Single-domain circular nanomagnets," Physical Review Letters, vol. 83, no. 5, pp. 1042-1045, 1999.

[10] T. Shinjo, T. Okuno, R. Hassdorf, K. Shigeto, and T. Ono, "Magnetic vortex core observation in circular dots of permalloy," Science, vol. 289, no. 5481, pp. 930-932, 2000.

[11] A. Wachowiak, J. Wiebe, M. Bode, O. Pietzsch, M. Morgenstern, and R. Wiesendanger, "Direct observation of internal spin structure of magnetic vortex cores," Science, vol. 298, no. 5593, pp. 577-580, 2002.

[12] D. Miu, S. I. Jinga, B. S. Vasile, and L. Miu, "Out of plane superferromagnetic behavior of quasi two-dimensional $\mathrm{Fe} / \mathrm{Al}_{2} \mathrm{O}_{3}$ multilayer nanocomposites," Journal of Applied Physics, vol. 117, no. 7, Article ID 074303, 2015.

[13] M. Varón, M. Beleggia, T. Kasama et al., "Dipolar magnetism in ordered and disordered low-dimensional nanoparticle assemblies," Scientific Reports, vol. 3, article 1234, 2013.

[14] A. P. Boltaev, F. A. Pudonin, and I. A. Sherstnev, "Vortex-like magnetization of multilayer magnetic nanoisland systems in weak magnetic fields," Applied Physics Letters, vol. 102, no. 14, Article ID 142404, 2013.

[15] X. Yan and Y. Xu, "Negative remanence in magnetic nanostructures," Journal of Applied Physics, vol. 79, no. 8, pp. 6013-6015, 1996.

[16] N. N. Kovaleva, D. Chvostova, A. V. Bagdinov et al., "Interplay of electron correlations and localization in disordered $\beta$-tantalum films: evidence from dc transport and spectroscopic ellipsometry study," Applied Physics Letters, vol. 106, no. 5, Article ID 051907, pp. 1-5, 2015.

[17] A. P. Boltaev, F. A. Pudonin, and I. A. Sherstnev, "Specific features of the magnetoresistance in multilayer systems of magnetic nanoislands in weak magnetic fields," Physics of the Solid State, vol. 53, no. 5, pp. 950-956, 2011. 
[18] I. A. Sherstnev, Electronic transport and magnetic structure of nanosland ferromagnetic materials systems [Ph.D. thesis], 2014, http://www.lebedev.ru/ru/dissertationcouncils/vak.html?date= 2014-04-28.

[19] R. Oja, M. Tyunina, L. Yao et al., " $d{ }^{0}$ ferromagnetic interface between nonmagnetic perovskites," Physical Review Letters, vol. 109, no. 12, Article ID 127207, pp. 1-5, 2012.

[20] J. Du, B. Zhang, R. K. Zheng, and X. X. Zhang, "Memory effect and spin-glass-like behavior in Co-Ag granular films," Physical Review B-Condensed Matter and Materials Physics, vol. 75, no. 1, Article ID 014415, pp. 1-7, 2007.

[21] N. N. Kovaleva, K. I. Kugel, A. V. Bazhenov et al., "Formation of metallic magnetic clusters in a Kondo-lattice metal: evidence from an optical study," Scientific Reports, vol. 2, article 890, pp. $1-7,2012$.

[22] N. N. Kovaleva, A. V. Boris, C. Bernhard et al., "Spin-controlled Mott-Hubbard bands in $\mathrm{LaMnO}_{3}$ probed by optical ellipsometry," Physical Review Letters, vol. 93, no. 14, Article ID 147204, pp. 1-4, 2004.

[23] N. N. Kovaleva, A. V. Boris, P. Yordanov et al., "Optical response of ferromagnetic $\mathrm{YTiO}_{3}$ studied by spectral ellipsometry," Physical Review B-Condensed Matter and Materials Physics, vol. 76, no. 15, Article ID 155125, pp. 1-11, 2007. 

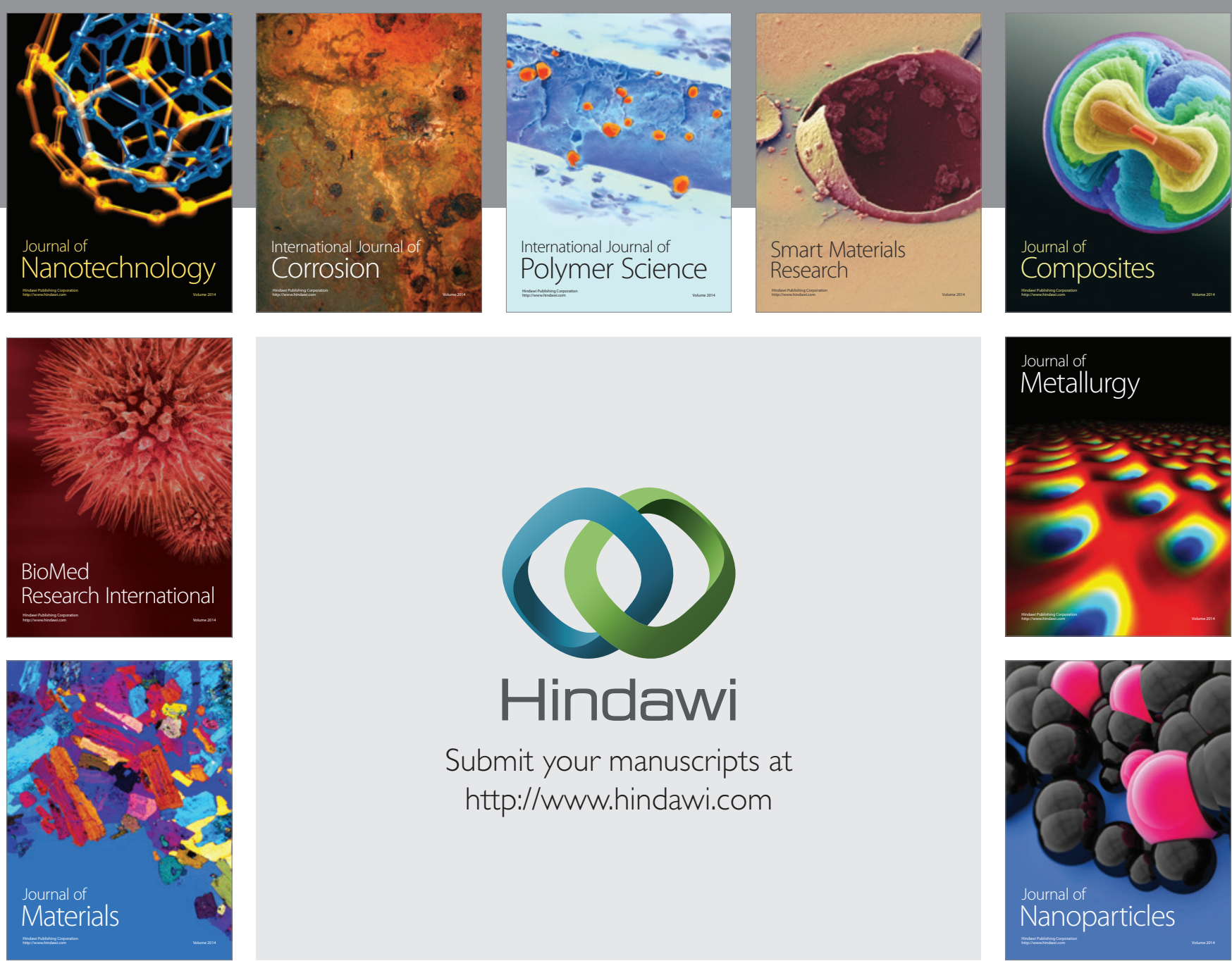

\section{Hindawi}

Submit your manuscripts at

http://www.hindawi.com

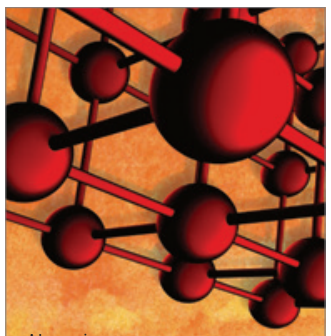

Materials Science and Engineering
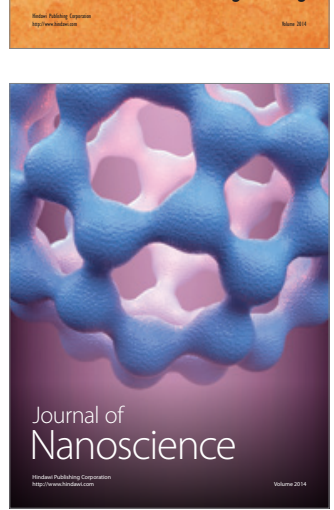
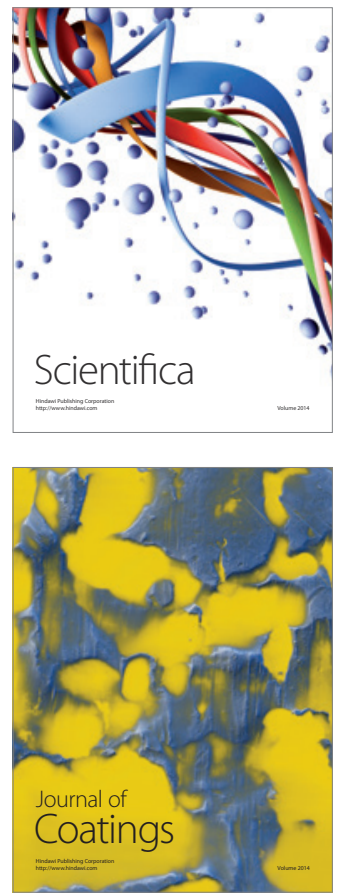
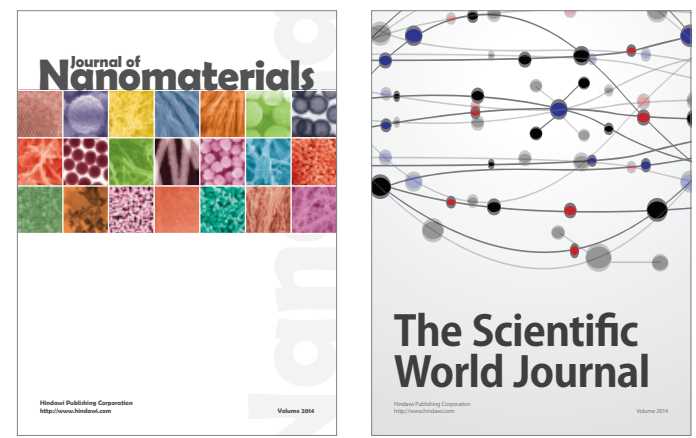

The Scientific World Journal
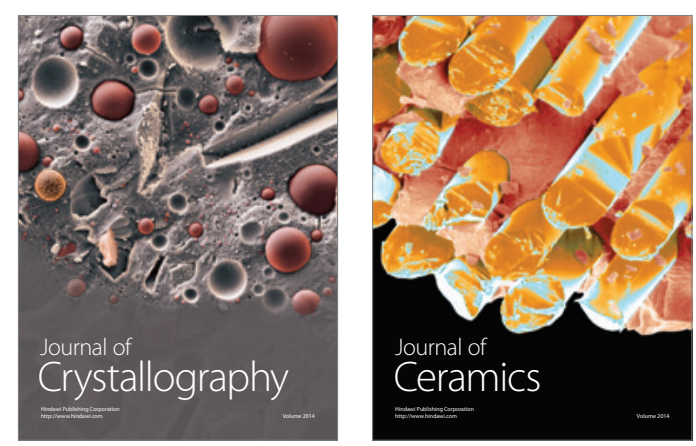
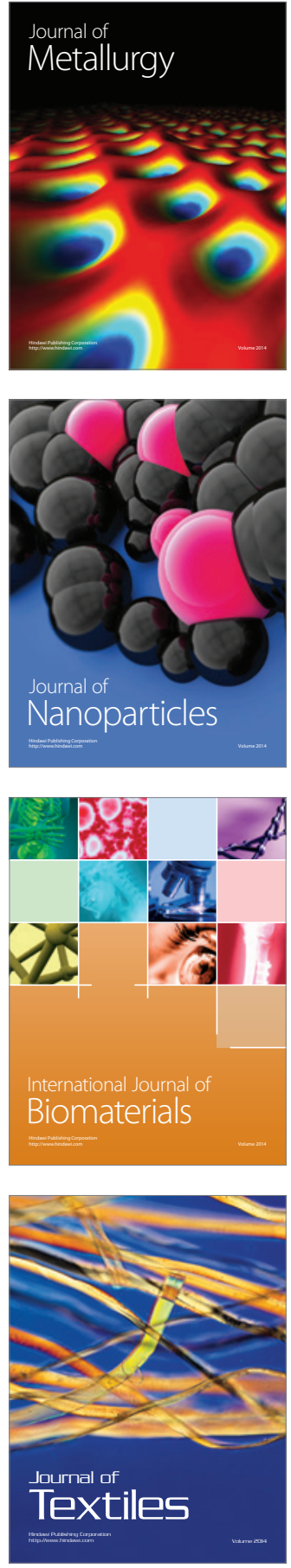